\title{
Boltzmann Entropy for the Spatial Information of Raster Data
}

\author{
Peichao Gao ${ }^{\mathrm{a},} *$, Hong Zhang ${ }^{\mathrm{b}}$, Zhilin $\mathrm{Li}^{\mathrm{c}}$ \\ ${ }^{a}$ Faculty of Geographical Science, Beijing Normal University, Beijing, China; gaopc@bnu.edu.cn \\ ${ }^{b}$ Faculty of Geosciences \& Environmental Engineering, Southwest Jiaotong University, Chengdu, Sichuan, China; \\ zhangh2011@swjtu.edu.cn \\ ${ }^{c}$ Department of Land Surveying and Geo-Informatics, The Hong Kong Polytechnic University, Kowloon, Hong Kong; \\ zl.li@polyu.edu.hk
}

* Corresponding author

Keywords: Boltzmann Entropy; Spatial Information; Raster Data; Spatial Data

\begin{abstract}
:
Entropy is an important concept that originated in thermodynamics. It is the subject of the famous Second Law of Thermodynamics, which states that "the entropy of a closed system increases continuously and irrevocably toward a maximum" (Huettner 1976, 102) or "the disorder in the universe always increases" (Framer and Cook 2013, 21). Accordingly, it has been widely regarded as an ideal measure of disorder. Its computation can be theoretically performed according to the Boltzmann equation, which was proposed by the Austrian physicist Ludwig Boltzmann in 1872. In practice, however, the Boltzmann equation involves two problems that are difficult to solve, that is the definition of the macrostate of a system and the determination of the number of possible microstates in the microstate. As noted by the American sociologist Kenneth Bailey, "when the notion of entropy is extended beyond physics, researchers may not be certain how to specify and measure the macrostate/microstate relations" (Bailey 2009, 151). As a result, this entropy (also referred to as Boltzmann entropy and thermodynamic entropy) has remained largely at a conceptual level.
\end{abstract}

In practice, the widely used entropy is actually proposed by the American mathematician, electrical engineer, and cryptographer Claude Elwood Shannon in 1948, hence the term Shannon entropy. Shannon entropy was proposed to quantify the statistical disorder of telegraph messages in the area of communications. The quantification result was interpreted as the information content of a telegraph message, hence also the term information entropy. This entropy has served as the cornerstone of information theory and was introduced to various fields including chemistry, biology, and geography. It has been widely utilized to quantify the information content of geographic data (or spatial data) in either a vector format (i.e., vector data) or a raster format (i.e., raster data). However, only the statistical information of spatial data can be quantified by using Shannon entropy. The spatial information is ignored by Shannon entropy; for example, a grey image and its corresponding error image share the same Shannon entropy.

Therefore, considerable efforts have been made to improve the suitability of Shannon entropy for spatial data, and a number of improved Shannon entropies have been put forward. Rather than further improving Shannon entropy, this study introduces a novel strategy, namely shifting back from Shannon entropy to Boltzmann entropy. There are two advantages of employing Boltzmann entropy. First, as previously mentioned, Boltzmann entropy is the ideal, standard measure of disorder or information. It is theoretically capable of quantifying not only the statistical information but also the spatial information of a data set. Second, Boltzmann entropy can serve as the bridge between spatial patterns and thermodynamic interpretations. In this sense, the Boltzmann entropy of spatial data may have wider applications. In this study, Boltzmann entropy is employed to quantify the spatial information of raster data, such as images, raster maps, digital elevation models, landscape mosaics, and landscape gradients. To this end, the macrostate of raster data is defined, and the number of all possible microstates in the macrostate is determined. To demonstrate the usefulness of Boltzmann entropy, it is applied to satellite remote sensing image processing, and a comparison is made between its performance and that of Shannon entropy. 